\title{
La obesidad y el síndrome metabólico como problema de salud pública. Una reflexión
}

\author{
Grupo académico para el estudio, la prevención y el tratamiento de la obesidad \\ y el síndrome metabólico de la Comisión Coordinadora de los Institutos Nacionales de Salud, \\ Hospitales Federales de Referencia y Hospitales de Alta Especialidad*
}

\section{Antecedentes}

La obesidad y el síndrome metabólico son entidades clínicas complejas y heterogéneas con un fuerte componente genético, cuya expresión está influida por factores ambientales, sociales, culturales y económicos, entre otros.

El incremento paralelo de la frecuencia de la obesidad y del síndrome metabólico es un fenómeno mundial y México no es la excepción. Aunado a esto, estas patologías son factores de riesgo importantes para el desarrollo de diabetes tipo 2, la enfermedad arterial coronaria y cerebrovascular por arteriosclerosis, que son las principales causas de muerte en nuestro país. El control de estas alteraciones metabólicas incide directamente en la morbi-mortalidad de muchos padecimientos; sin embargo, en la actualidad no existen estrategias de prevención, diagnóstico y tratamiento eficaces para la mayoría de los casos. Por estas razones, la obesidad y el síndrome metabólico se han convertido en un serio problema de salud pública en los países occidentalizados.
En los últimos años ha crecido el interés de investigadores y clínicos de distintas disciplinas en el estudio de la obesidad y del síndrome metabólico. Como es frecuente en las enfermedades complejas, la visión de los expertos tiene una perspectiva limitada y en el peor de los casos, excluyente de otras que son complementarias. Si no se trata de un problema de salud pública, esta situación podría resultar deseable en aras de la pureza de los procesos de generación de conocimiento. Sin embargo, dada la relevancia de estos padecimientos en la salud de la comunidad se requiere encontrar estrategias científicas que acorten los tiempos en la generación de conocimientos y que permitan diseñar modelos de prevención y tratamiento. La meta se alcanzará cuando estos modelos sean operables a través de programas asistenciales y se logre disminuir la frecuencia de estas entidades.

En las últimas dos décadas la información sobre obesidad y síndrome metabólico ha crecido rápidamente, lo que ha llevado a algunos sistemas de salud en el mundo y a numerosas sociedades científicas a formar

Por considerarlo de interés y con la autorización correspondiente, el Consejo de Editores de Salud Pública de México determinó conveniente incluir este trabajo, el cual se publicó previamente en el número 4 del volumen 29 de la revista Acta Pediátrica de México. El documento se publicó también en los volúmenes de 2008 de las revistas Salud Mental y Archivos de Cardiología.

* Eduardo García-García, M Esp, (I) Manuel De la Llata-Romero, M Esp, (2) Martha Kaufer-Horwitz, Dr en CS,(I) María Teresa Tusié-Luna, D en Gen,(I) Raúl Calzada-León, M Esp, ${ }^{(3)}$ Verónica Vázquez-Velázquez, Psicol, (I) Simón Barquera-Cervera, $\mathrm{PhD}$, ${ }^{(4)}$ Alejandro de Jesús Caballero-Romo, M Esp, ${ }^{(5)}$ Lorena Orozco, DC, ${ }^{(6)}$ DavidVelásquez-Fernández, D en CM, ${ }^{(6)}$ Martín Rosas-Peralta, M Esp, ${ }^{(7)}$ Armando Barriguete-Meléndez, M Esp, ${ }^{(1)}$ Rogelio Zacarías-Castillo, M Esp, ${ }^{(8)}$ Julio Sotelo-Morales, M Esp. ${ }^{(2)}$

(I) Instituto Nacional de Ciencias Médicas y Nutrición Salvador Zubirán

(2) Comisión Coordinadora de los Institutos Nacionales de Salud, Hospitales Federales de Referencia y Hospitales de Alta Especialidad

(3) Instituto Nacional de Pediatría

(4) Instituto Nacional de Salud Pública

(5) Instituto Nacional de Psiquiatría Ramón de la Fuente Muñiz

(6) Instituto Nacional de Medicina Genómica

(7) Instituto Nacional de Cardiología Ignacio Chávez

(8) Hospital General Dr. Manuel Gea González 
grupos de expertos que analicen esta información de manera permanente. En México los esfuerzos que se realizan por los Institutos Nacionales de Salud, Hospitales Federales de Referencia y Hospitales de Alta Especialidad para el desarrollo de investigación básica y clínica de la obesidad, son numerosos; sin embargo, la difusión de los resultados y la comunicación entre los investigadores aún no es suficiente. Se carece de un plan maestro que señale logros alcanzados y tareas por desarrollar. Esto ha determinado que la Comisión Coordinadora de Institutos Nacionales de Salud y Hospitales de Alta Especialidad (CCINSHAE) haya propuesto la formación de un Grupo Académico para el Estudio la Prevención y el Tratamiento de la Obesidad y Síndrome Metabólico. El Grupo elaboró un documento que pretende reunir las reflexiones y las propuestas generales de este grupo de investigadores y clínicos de los Institutos Nacionales de Salud, Hospitales Federales de Referencia y Hospitales de Alta Especialidad. Este es el primer paso para colaborar en el desarrollo de objetivos y lineamientos comunes en el sector salud, con el propósito de comprender y así poder combatir la obesidad y el síndrome metabólico.

\section{¿Por qué analizar en conjunto la obesidad} y el síndrome metabólico?

El análisis de la obesidad y el síndrome metabólico en conjunto se ha venido construyendo desde distintos escenarios y obedece a distintos objetivos, necesidades e intereses. Por ello no hay una respuesta simple a esta pregunta y probablemente todo intento por obtener una respuesta definitiva resulte insuficiente. En las distintas definiciones del síndrome metabólico del adulto dadas por Panel de Tratamiento para el Adulto III (ATP-III), la Organización Mundial de la Salud (OMS) y la Federación Internacional de la Diabetes (IDF), el parámetro más constante como criterio diagnóstico es la obesidad, evaluada por el índice de masa corporal (IMC: $\geq 30 \mathrm{~kg} / \mathrm{m}^{2}$ ) y el perímetro de cintura: mujeres: $>88 \mathrm{~cm}$; hombres, $>102 \mathrm{~cm} .{ }^{1}$ En población pediátrica, la IDF ha propuesto que para sospechar una entidad similar al síndrome metabólico, debe existir obesidad, es decir, IMC mayor a la centila 95 más dos criterios adicionales (cuadro I).

El termino "síndrome metabólico" agrupa varios factores de riesgo cardiovascular, el principal de los cuales es la resistencia a la acción de la insulina. Sin embargo la fisiopatogenia del síndrome metabólico, la obesidad parece ser uno de los factores desencadenantes más importantes entre otras alteraciones metabólicas que lo caracterizan: intolerancia a la glucosa, diabetes, dislipidemia e hipertensión. Por lo tanto, en la mayoría

\section{Cuadro I Definiciones de Síndrome Metabólico}

En población adulta

a) Organización Mundial de la Salud

Dos o más de los siguientes criterios:

I. Hipertensión arterial (140/90)

2. Hipertrigliceridemia $(>150 \mathrm{mg} / \mathrm{dL})$ y/o colesterol $\mathrm{HDL}<35 \mathrm{mg} / \mathrm{dL}$ en hombres $0<40$ en mujeres

3. Microalbuminuria $>20 \mathrm{microgramos} / \mathrm{min}$

4. Obesidad: $I M C>29.9 \mathrm{~kg} / \mathrm{m}^{2}$ y/o relación cintura/cadera elevada (hombres $>0.9$, mujeres $>0.85$ )

Más la presencia de una de las siguientes condiciones:

Diabetes tipo 2, intolerancia a la glucosa o resistencia a la insulina (insulina de ayuno > percentil 75 de la población en estudio)

b) Programa Nacional de Educación en Colesterol (ATP III)

Tres o más de las siguientes condiciones:

I. Obesidad: Perímetro de cintura $>88 \mathrm{~cm}$ en mujeres y $>102 \mathrm{~cm}$ en hombres.

2. Hipertrigliceridemia: $>150 \mathrm{mg} / \mathrm{dL}$.

3. Colesterol HDL bajo: $<40 \mathrm{mg} / \mathrm{dL}$ en hombres $y<50$ en mujeres.

4. Hipertensión arterial: $130 / 85 \mathrm{mmHg}$ o diagnóstico previo.

5. Diabetes o glucosa anormal de ayuno: $100 \mathrm{mg} / \mathrm{dL}$.

c) Federación Internacional de Diabetes (IDF)

Obesidad central: perímetro de cintura $\geq 94 \mathrm{~cm}$ en hombres $y \geq 80 \mathrm{~cm}$ en mujeres (de origen europeo); con valores de especificidad étnica para otros grupos.

Además, dos de los siguientes cuatro factores:

I. Hipertrigliceridemia: $\geq 150 \mathrm{mg} / \mathrm{dL}$ [ $1.7 \mathrm{mmol} / \mathrm{L}]$, o tratamiento específico para esta anormalidad.

2. Colesterol HDL bajo: (< $40 \mathrm{mg} / \mathrm{dL}[1.0 \mathrm{mmol} / \mathrm{L}]$ en hombres $y<50 \mathrm{mg} / \mathrm{dL}$ [ $1.3 \mathrm{mmol} / \mathrm{L}]$ en mujeres o tratamiento específico para esta anormalidad.

3. Presión arterial alta: sistólica $\geq 130$ ó diastólica $\geq 85 \mathrm{~mm} \mathrm{Hg} \circ$ tratamiento para hipertensión previa.

4. Diabetes previa o glucosa anormal en ayuno: $\geq 100 \mathrm{mg} / \mathrm{dL}[5.6 \mathrm{mmol} / \mathrm{L}]$. Si la glucosa en ayuno es superior a $5.6 \mathrm{mmol} / \mathrm{L} \circ 100 \mathrm{mg} / \mathrm{dL}$, se recomienda una carga de tolerancia a la glucosa oral aunque no es necesaria para definir la presencia del síndrome.

\section{En población pediátrica}

a) Federación Internacional de Diabetes (IDF)

Obesidad: IMC mayor a la centila 95 para una población dada.

Además, dos o más de los siguientes criterios:

I. Prepúberes: Cintura mayor a la centila 90

2. Púberes: Cintura mayor a la centila 90

a. Triglicéridos $>$ a centila 90

b. HDL-C $<$ a la centila 10

c. $\mathrm{TA}>130 / 85$

d. Glucosa en ayuno $>100 \mathrm{mg} / \mathrm{dL}$

3. Postpúberes: Cintura $>94 \mathrm{~cm}$. en varones

a. Cintura $>80 \mathrm{~cm}$. en mujeres

b. $\mathrm{HDL}<40 \mathrm{mg} / \mathrm{dL}$ en varones

c. $\mathrm{HDL}<50 \mathrm{mg} / \mathrm{dL}$ en mujeres

d. $\mathrm{TA}>130 / 85$

e. Glucosa en ayunas $>100 \mathrm{mg} / \mathrm{dL}$

Fuentes: Referencias 2, 3,4,5 
de los casos la expresión del síndrome metabólico ocurre en individuos obesos. En muchos casos, la expresión del síndrome metabólico es en buena medida una comorbilidad de la obesidad.

La obesidad y el síndrome metabólico como fenómenos independientes, hacen necesario identificar los mecanismos responsables de la asociación entre estas condiciones. Es importante comprender los mecanismos del síndrome metabólico que propician la enfermedad en individuos no obesos; en el caso de la obesidad, importa dilucidar los que los protegen contra alteraciones metabólicas. En la actualidad, estos problemas son motivo de numerosas investigaciones que aún no han generado respuestas definitivas.

Sin restar importancia a lo anterior, en la práctica clínica es muy útil asociar estas dos condiciones para fines de prevención, diagnóstico y tratamiento, por las siguientes razones:

1. Numerosos estudios han demostrado que se reduce hasta 70\% la expresión clínica del síndrome metabólico en el paciente obeso que logra buen resultado con el tratamiento de su obesidad. Aunque no hay estudios longitudinales para saber si la corrección de la hipertensión arterial, la hiperglucemia y la dislipidemia reducen el riesgo de eventos cardiovasculares o de muerte, se espera que estos cambios metabólicos favorables se acompañen de reducción de los llamados eventos finales.

2. Las medidas generales de prevención y tratamiento de ambas condiciones tienen numerosas coincidencias. En el tratamiento de la obesidad y del síndrome metabólico es indispensable que el paciente cambie su estilo de vida por conductas más saludables, especialmente aumentando su actividad física, reducción del consumo de la energía total y disminuyendo el consumo de grasas y alcohol.

3. Para el manejo de la obesidad los médicos y nutriólogos deben tomar en cuenta sus comorbilidades. No ha sido fácil la transición que tiene que realizar el personal en salud sobre los conocimientos acerca de los padecimientos crónico-degenerativos.

4. La infraestructura para el diagnóstico y tratamiento de estos padecimientos, así como los recursos humanos que realizan estos procesos son esencialmente los mismos.

La obesidad, a diferencia de otras enfermedades como las infecciones, el cáncer y las enfermedades mentales, es una enfermedad progresiva que puede revertirse o controlarse más fácilmente en su fase inicial. La detección y tratamiento de comorbilidades en el paciente obeso permite construir una relación médico-paciente más adecuada, ya que en la medida que el médico, el nutriólogo o cualquier otro profesional de la salud detecta la presencia de una enfermedad y el paciente toma conciencia de ella, se favorece la adherencia al tratamiento.

Desde la perspectiva de la salud pública asociar ambas entidades permite transmitir a la comunidad varios mensajes más fácilmente:

a) Reforzar la noción de que la obesidad es una enfermedad.

b) Transmitir el concepto de que existe "un continuum" entre la obesidad y el síndrome metabólico.

c) Transmitir la importancia de controlar la obesidad para evitar o retardar la expresión de comorbilidades, en particular del síndrome metabólico.

d) Hacer énfasis en que la detección de la obesidad -en particular la abdominal-permite identificar un mayor número de individuos con riesgo de sufrir eventos isquémicos.

e) Acudir periódicamente a los sistemas de salud para el control y prevención de estos padecimientos.

Por las razones anteriores, analizar la obesidad y el síndrome metabólico en conjunto parece ser el modelo más ventajoso desde el punto de vista de la salud pública y del manejo integral del paciente. El análisis en conjunto tiene algunos inconvenientes que resultan menores frente a las ventajas descritas.

La obesidad y el sindrome metabólico como problema de salud

La obesidad puede considerarse como el problema de salud pública principal al cual se enfrenta México en la actualidad dada su gran prevalencia, sus consecuencias y su asociación con las principales causas de mortalidad.

La salud pública juega un papel fundamental tanto en la identificación de factores determinantes y sus posibles soluciones como en la implementación de medidas poblacionales para su control y la evaluación de su eficacia.

En los últimos seis años la prevalencia de sobrepeso y obesidad en el adulto ha aumentado 12\% (ENSANUT, 2006) y tiene un patrón muy similar al de los mexicanos que viven en los Estados Unidos, que es uno de los grupos con mayor prevalencia, cercana a $70 \%$ tanto en hombres como mujeres. El problema es igualmente alarmante en niños y adolescentes.

Es necesario identificar con precisión los factores determinantes ambientales que contribuyen al problema con gran énfasis en la comprensión de los factores básicos y subyacentes, tales como acceso a alimentos 
saludables, entornos que fomenten la actividad física y al conocimiento de la población para el autocuidado y los mecanismos involucrados en estos procesos.

La capacitación adecuada del sector médico es fundamental para contribuir al control de la obesidad y el sobrepeso. Importa reconocer que se pueden lograr grandes cambios con implementación de políticas orientadas a abatir los determinantes ambientales más importantes. Por ejemplo, la generación de entornos seguros y adecuados para que la población, y particularmente las mujeres, puedan desarrollar actividad física; la promoción intensiva y orientación desde el entorno escolar sobre alimentación saludable y actividad física; incentivos para desarrollos comunitarios que promuevan la actividad física, etcétera.

El consumo de bebidas que proporcionan energía como jugos, aguas frescas, refrescos, te o café con azúcar, bebidas endulzadas, leche entera y bebidas alcohólicas, eleva considerablemente la ingestión energética promedio en los mexicanos. Dependiendo del grupo de edad, entre 19 y $22 \%$ de la energía de la dieta proviene de bebidas. En los niños, la leche entera es la bebida que más contribuye a la ingestión energética, por lo cual es importante promover programas sociales de abasto de leche a comunidades de bajos recursos, con alternativas al consumo de leche entera, en virtud de que la obesidad infantil ya no es un problema exclusivo de los grupos de mayor nivel socioeconómico. También, promover el consumo de leche baja en grasa como una alternativa saludable especialmente para niños con sobrepeso o con riesgo de tenerlo. En adultos es necesario orientarles sobre la importancia de consumir bebidas sin energía como agua simple para hidratarse.

Numerosas intervenciones de carácter poblacional tienen el potencial de contribuir al control del problema de la obesidad y sobrepeso. Entre éstas, las intervenciones en el entorno escolar, los espacios laborales y grandes grupos profesionales, como los maestros, trabajadores de sectores, etc. Este es uno de los grandes retos de la salud pública en el problema de la obesidad.

La evidencia de intervenciones exitosas para la prevención y el control de la obesidad a nivel poblacional, por ejemplo en el entorno escolar o en espacios laborales, se encuentra disponible; lo que hace falta es una traducción efectiva del conocimiento a políticas de salud pública y su implementación.

El área de regulación e incentivos a la industria y el sector productivo es una de las que mayor oportunidad tiene de identificar modificaciones de bajo costo con gran impacto. Entre éstas se han propuesto la promoción del consumo de agua pura y apoyos para que los grandes grupos industriales promuevan alimentación correcta y actividad física en sus empleados, entre otras estrategias.

Los tres grandes objetivos de la salud pública son la prevención primaria, la prevención secundaria y el control del daño; su papel en el problema de la obesidad debe estar claramente identificado. Los Institutos Nacionales de Salud pueden contribuir a ello y a lograr que los planes de salud incluyan los objetivos correspondientes. No basta con reconocer que es necesaria la prevención de la obesidad ya que millones de mexicanos ya la padecen. Por lo general estos son adultos que toman decisiones sobre la alimentación de sus familias. Se requieren buenas estrategias para el tratamiento, el control y la prevención de complicaciones. Hay que reconocer que se requiere gran creatividad para abordar este problema e identificar posibles soluciones.

En el campo de la educación, la promoción y la mercadotecnia social, se dan diversos mensajes de prevención comunes, como la leyenda "coma frutas y verduras" que no han mostrado tener el impacto necesario. La comunicación de mensajes a la población de forma efectiva no depende únicamente de la identificación de los problemas y el conocimiento de sus determinantes sino también del uso de técnicas de persuasión similares a las utilizadas por la industria, lo cual requiere inversión y asociación de expertos en el área a los equipos de promoción. Las campañas y "slogans" intuitivos hechos por personal de la salud, así como toda idea, deben ser considerados sustrato para que grupos de creativos especializados generen las comunicaciones finales dirigidas a la población. Sólo así se lograría una calidad e impacto similares a la que consigue la publicidad enfocada a la venta de productos.

Sindrome Metabólico: más allá de la concatenación de factores de riesgo

En el mundo cada cuatro segundos ocurre un infarto agudo del miocardio y cada cinco segundos un evento vascular cerebral. En México, en la población adulta (20 a 69 años) hay más de 17 millones de hipertensos, más de 14 millones de dislipidémicos, más de 6 millones de diabéticos, más de 35 millones de adultos con sobrepeso u obesidad y más de 15 millones con grados variables de tabaquismo. ${ }^{6}$ En América Latina $75 \%$ de la mortalidad total en adultos se debe a enfermedades crónicas. ${ }^{7}$

Nuestra pirámide poblacional muestra que la mayoría de los adultos (75\%) tiene menos de 55 años de edad y aunque la prevalencia de los factores de riesgo cardiovascular es mayor después de los 40 años, en datos absolutos, los millones de portadores de estos factores de riesgo corresponden a la población económicamente 
activa, por lo que sus consecuencias socioeconómicas y en la calidad de vida. De ahí que pueden ser devastadoras las afecciones cardiovasculares que caen dentro del rubro de gastos catastróficos. ${ }^{8}$

Ante el crecimiento en el conocimiento de las ciencias básicas, el clínico ha centrado su atención en favorecer las buenas prácticas clínicas a través del desarrollo de ensayos clínicos controlados, con un alto rigor científico, que permitan normar las mejores guías para la práctica clínica. ${ }^{9}$

Nuestro descenso en el pensamiento clínico integral ha permitido que una entidad nosológica sea vista en su simple entorno de "existe o no existe", "la tiene o no la tiene", basándonos en puntos de corte arbitrarios. ${ }^{10}$

Hoy día, todo mundo habla de las enfermedades crónicas no transmisibles, como la hipertensión, la diabetes, las dislipidemias y la obesidad entre otras, responsables de generar enfermedad vascular con afección a órganos blanco. La aterotrombosis es la vía final más común, responsable, entre otras, de la cardiopatía isquémica, que es la causa número uno de muerte en el adulto. ${ }^{11}$ Pero también de otras enfermedades devastadoras como la insuficiencia renal, cerebral, ceguera e incluso cáncer.

En el ámbito cardiovascular, un principal serio problema con el síndrome metabólico es la estimación del pronóstico a partir de valores un tanto arbitrarios. Es decir, parecería que tener síndrome metabólico es mucho más peligroso que tener una presión arterial de 280/160 mm Hg o 600 mg/dL de glucosa u 800 mg/dL de colesterol. ${ }^{12}$

En este contexto, el síndrome metabólico debe ser interpretado como una "concatenación de factores de riesgo cardiovascular", donde el principal mensaje debe ser que, ante todo paciente que tenga un factor de riesgo, siempre debe tenerse en mente la posibilidad de que haya otro u otros factores de riesgo cardiovascular, sobre todo si se es obeso y mayor de 30 años. ${ }^{13}$

\section{¿Qué se está haciendo en los Institutos?}

A pesar de la alta prevalencia de obesidad en México, la mayoría de los Institutos Nacionales de Salud, Hospitales Federales de Referencia y Hospitales de Alta Especialidad no tienen clínicas dedicadas a la atención del paciente obeso. Se exceptúa la Clínica de Obesidad del Instituto Nacional de Ciencias Médicas y Nutrición Salvador Zubirán (INCMNSZ).

En la mayoría de las instituciones, distintos servicios realizan esfuerzos aislados, en asistencia e investigación; sin embargo, no existen lineamientos, programas o abordajes multidisciplinarios coordinados dentro de las instituciones ni entre ellas.
Algunas instituciones están más enfocadas a la atención del problema en sí mismo (INCMNSZ, Instituto Nacional de Pediatría -INP); otras se concentran en las complicaciones crónicas del problema (por ejemplo, Instituto Nacional de Enfermedades Respiratorias (INER), Instituto Nacional de Cardiología Ignacio Chávez (INCICh), Instituto Nacional de Cancerología (INCAN), Instituto Nacional de Perinatología Isidro Espinoza de los Reyes (INPerIER), INCMNSZ), mientras que el Instituto Nacional de Salud Públcia (INSP) se aboca a aspectos epidemiológicos y a la definición de los determinantes ambientales de estas entidades.

Existe la necesidad de generar conocimiento a través de la investigación tanto para crear nuevos modelos de atención y tratamiento como para identificar los mecanismos celulares y moleculares implicados en la morbimortalidad del paciente con obesidad y síndrome metabólico. Es necesario coordinar un esfuerzo de investigación que integre distintas instituciones y capacite recursos humanos para estas tareas.

Los esfuerzos que realizan de manera aislada médicos e investigadores en los distintos institutos y hospitales de alta especialidad desarrollando líneas de investigación en este campo no son suficientes, ni eficazmente aprovechados. En algunos sitios como el INSP o el INCMNSZ existen numerosos grupos y proyectos de investigación; sin embargo, la difusión y el impacto que de ellos deriven siempre será limitado mientras no exista una estrecha coordinación y colaboración interinstitucional.

Llama la atención por ejemplo, que a pesar de los presupuestos reducidos para la atención de la obesidad y sus comorbilidades, algunos hospitales de segundo nivel de atención ya están realizando cirugía bariátrica y reconstructiva en pacientes con obesidad mórbida. Esta situación obliga a generar políticas y lineamientos claros en coordinación con las instituciones que integran la CCINSHAE.

Instrumentos de los que disponemos: normas y guías. Su aplicabilidad

Hay numerosos documentos de distintos grupos que abordan el tema de la obesidad y el síndrome metabólico de manera directa o indirecta. La Secretaría de Salud ha emitido once Normas Oficiales Mexicanas que de alguna manera se relacionan con el tema en cuestión. ${ }^{14}$ Por otra parte, la Sociedad Mexicana de Nutrición y Endocrinología ha publicado varios consensos sobre la obesidad y el síndrome metabólico. También existen documentos emitidos por grupos de Estados Unidos, de Canadá y de organismos internacionales que buscan consensos en la identificación, diagnóstico, tratamiento 
y prevención de estas entidades. Esta información debe ser analizada a fin de establecer puntos de acuerdo entre las distintas instituciones de salud en torno a un abordaje homogéneo del problema que permita en el corto y mediano plazos contrastar y evaluar los esfuerzos que se hacen para responder a la situación actual de la obesidad y sus comorbilidades.

\section{La salud como un patrimonio individual}

La pérdida de la salud conlleva una serie de alteraciones en distintos ámbitos. En particular, las enfermedades crónicas se caracterizan por un deterioro progresivo como consecuencia de complicaciones discapacitantes o mortales. El individuo enfermo es menos productivo y con ello contribuye al detrimento paulatino de la economía familiar, tanto por el ausentismo laboral y eventualmente el desempleo, como por el incremento en el gasto familiar derivado de su tratamiento.

La OMS define a la salud como el estado de bienestar físico, psicológico y social del individuo que permite un desarrollo armónico del mismo. De acuerdo con esta definición la salud debe ser considerada un patrimonio individual, esto es, un bien único que debe preservarse.

En este sentido las acciones de gobierno y la sociedad deben orientarse a la prevención de enfermedades comunes con características de epidemia, como la obesidad y la diabetes. Sin embargo, ¿Cómo prevenir y controlar una enfermedad que no es considerada como una entidad patológica por sí misma?

La obesidad es una alteración metabólica que se asocia a enfermedades graves como la diabetes tipo 2, la hipertensión arterial y la enfermedad arterial coronaria. A pesar de que la obesidad es uno de los principales factores de riesgo para estas entidades, rara vez se le considera como una enfermedad por sí misma. Algunas razones para esta apreciación son: 1) la variedad y complejidad de las causas que la originan; 2) el escaso conocimiento sobre los mecanismos fisiopatológicos implicados; 3) la alta prevalencia de obesidad entre la población infantil y adulta, lo cual lleva a percibir a esta entidad como una característica "frecuente o común" y 4) la poca información que tiene la población general sobre los efectos adversos de la obesidad.

La obesidad es un fenómeno tan complejo que su prevención y control requieren esfuerzos coordinados para entender y tratarla con éxito. La magnitud del problema es $\tan$ grave, que a pesar de que hay aproximadamente 18 millones de obesos en nuestro país, en los centros de salud se atiende por esta condición sólo a una pequeña fracción, esto es, al obeso que busca atención médica. Aún más, esta atención se otorga a través de modelos de atención relativamente nuevos, no probados y con poco éxito en lo que respecta a costos y adherencia.

Por otro lado, si la mayoría de los pacientes obesos buscaran atención médica el sistema de salud sería insuficiente en relación a la infraestructura, a los recursos humanos y a los gastos de operación. Es por ello prioritario generar modelos de atención en el paciente obeso orientados a la prevención de sus complicaciones.

\section{Prevención de la obesidad y del síndrome metabólico}

La alta prevalencia de la obesidad es una realidad en los países industrializados y en los que están en vías de desarrollo. La información disponible indica un aumento acelerado del problema que de no contenerse, puede tener repercusiones importantes en los indicadores de salud de muchos países incluyendo el nuestro. Es preocupante que a pesar de la gran cantidad de investigaciones e intervenciones realizadas en otros países para prevenir y combatir la obesidad, particularmente en la infancia, aún no se cuenta con una estrategia idónea, aplicable a cualquier contexto.

La importancia de la prevención de la obesidad radica en su naturaleza de enfermedad incurable y en los riesgos que implica. Se ha sugerido que la obesidad debe tratarse de forma similar a otras enfermedades incurables como el alcoholismo y el tabaquismo, donde la eliminación total del alcohol y del tabaco es un aspecto fundamental del tratamiento. El manejo de la obesidad es más complejo debido a que no es posible eliminar los alimentos del entorno de la persona obesa.

La obesidad se desarrolla con el tiempo y una vez instalada es irreversible y difícil de tratar. Además, las consecuencias de la enfermedad se deben al estrés metabólico y físico ocasionado por el exceso de peso crónico. Las consecuencias de la obesidad, como las cardiopatías, la resistencia a la insulina, la diabetes mellitus tipo 2, entre otras, pueden no ser reversibles en algunos pacientes, incluso a pesar de la pérdida de peso. Por otra parte, México ocupa el segundo lugar de obesos en el mundo, así que desde un enfoque poblacional, los recursos destinados por el Estado son insuficientes para ofrecer tratamiento a todos los afectados. El alto costo socioeconómico de la obesidad y del síndrome metabólico, es la limitante más importante para lograr atención integral a nivel nacional. En otras palabras, si se previene la obesidad, se abatirán los costos de atención de pacientes con enfermedad cardiovascular o con diabetes que hoy día representan las primeras causas de morbilidad y mortalidad en adultos. De ahí la importancia de concentrar los recursos en actividades de prevención y promoción de la salud. 
Actualmente, una proporción elevada de la población infantil y adolescente en México tiene sobrepeso u obesidad (5.3\% de los menores de 5 años, 26\% de los escolares y más del $30 \%$ de los adolescentes, según datos de la ENSANUT 2006). De acuerdo a las tendencias que muestran las encuestas nacionales de los últimos años, la prevalencia va en aumento. Es indudable que el sobrepeso en la infancia es un factor de riesgo de obesidad en el adulto, con todo lo que esto implica respecto a comorbilidades que se magnifican al manifestarse desde etapas tempranas por tener evoluciones largas. En vista de lo anterior, la implementación de acciones efectivas de prevención desde la infancia debe ser una prioridad de nuestros Sistemas de Salud.

A partir de un riesgo basal no modificable debido a la carga genética, la expresión de la obesidad se da por la acumulación de factores de riesgo a lo largo del espectro de la vida. Algunas de las estrategias de prevención en cada una de las etapas vitales son las siguientes:

En la vida fetal: prevenir la nutrición materna inadecuada, sea deficiente o excesiva, y el bajo peso al nacimiento. En la infancia: promover la lactancia materna y los esquemas de ablactación adecuados y oportunos; prevenir las infecciones y la desnutrición proteíno-energética; vigilar la velocidad de crecimiento; promover la actividad física y los hábitos alimentarios correctos donde el consumo de frutas y verduras sea un elemento central y fomentar el desarrollo de una autoestima adecuada.

En la adolescencia: promover la actividad física y evitar el sedentarismo; promover hábitos alimentarios adecuados, en particular el consumo de frutas y verduras y prevenir el tabaquismo y el consumo de alcohol.

En la edad adulta: promover la vida activa y la alimentación correcta, prevenir el tabaquismo y el consumo excesivo de alcohol; promover la vigilancia de la salud: peso, tensión arterial, glucosa, lípidos sanguíneos, atender oportunamente las alteraciones de esta edad.

La OMS, a través de la Organización de las Naciones Unidas para la Alimentación y la Agricultura (FAO), así como numerosas investigaciones, han establecido que el elemento central en la génesis de la obesidad es el desequilibrio energético, es decir, se consume más energía de la que se gasta. De esto se desprende que una alimentación adecuada y el combate al sedentarismo son elementos centrales que deben formar parte de cualquier esquema de prevención.

De lo anterior surge una pregunta: ¿La responsabilidad en el mantenimiento de un peso corporal saludable y la consecuente prevención de la obesidad, es individual o compartida? Es claro que cada individuo decide lo que va a comer de acuerdo con sus posibilidades y si va a incrementar su actividad física. Así, los individuos podrían beneficiarse notablemente por cambios en el ambiente que les faciliten un estilo de vida saludable. De esta forma es posible apoyar las decisiones y el esfuerzo individual con programas de promoción de la salud y de educación. Los logros serán todavía mayores si al mismo tiempo se efectúan cambios ambientales que apoyen el consumo de dietas correctas y la vida activa. La bondad del enfoque ambiental radica y su cambio equivale a varias decisiones diarias por un gran número de individuos, donde se tiene un alcance mayor, más sostenible y con menor costo en el largo plazo. Esto es particularmente importante para comunidades de bajos recursos donde se concentra el riesgo de obesidad.

Dentro de esta responsabilidad compartida, la prevención de la obesidad debe darse en distintos niveles, desde el individual hasta el legislativo. El sistema de salud debe tener un papel crucial en la promoción de la salud y la prevención de la obesidad y del síndrome metabólico a través de la orientación al personal y a los usuarios en los distintos temas centrales como la promoción de lactancia materna, la vigilancia del crecimiento, la promoción de una alimentación correcta y de una vida activa.

La prevención debe ser una estrategia prioritaria de salud pública que debe iniciarse en la infancia, continuarse a lo largo de la vida y tener la participación activa y comprometida del personal de salud junto con otros sectores de la sociedad. Vale la pena insistir que cuanto más temprano sea su inicio, los beneficios a corto, mediano y largo plazo serán más importantes, evidentes y eficaces.

\section{Problemas de la práctica clínica}

El listado de los problemas que la obesidad y el síndrome metabólico plantean en la práctica clínica es grande, lo que deja en claro la necesidad de investigaciones y cambios en las estrategias de atención de estos padecimientos. En nuestra opinión los problemas más sobresalientes son:

1. Ausencia de un lenguaje y objetivos comunes entre el trabajador de la salud y el paciente. Las preocupaciones médicas frente a la obesidad y el síndrome metabólico están claramente definidas y sistematizadas. El proceso a través del cual se llegó a esta información validada ha sido prolongado y data al menos de las últimas dos décadas. Un número creciente de médicos y nutriólogos conocen estos objetivos; sin embargo, el entrenamiento para proponerlos al paciente y facilitar su realización es en general inadecuado. Médicos y nutriólogos hacen prescrip- 
ciones farmacológicas y nutricias respectivamente, sin tener, en general, estrategias de motivación y sin la visión de un programa psicoeducativo que facilite al paciente alcanzar los cambios de conducta que requiere para controlar su padecimiento. Por otra parte los pacientes tienen expectativas poco realistas: perder peso en poco tiempo y sin esfuerzo. Aceptan seguir una dieta y tomar fármacos pero con la expectativa de curación, para así poder regresar a sus hábitos. La noción de control como un proceso que requiere atención y conductas saludables por tiempo indefinido no resulta atractiva. En el caso concreto de la obesidad, con mucha frecuencia las expectativas son fundamentalmente estéticas y de aceptación por los otros -pareja, familia, grupo social.

2. Propuestas terapéuticas que surgen de conocimiento generado en fechas recientes, por grupos interdisciplinarios. Esto determina que en la actualidad la mayoría de médicos, nutriólogos, psicólogos y otros trabajadores de la salud no estén adecuadamente capacitados para comprender y contender con estos padecimientos. Los programas universitarios a nivel licenciatura no hacen el énfasis necesario en ellos.

3. Infraestructura inadecuada en la mayoría de los consultorios e instituciones. La infraestructura material que se requiere para atender adecuadamente a estos pacientes no es especializada ni particularmente costosa. A pesar de ello, hay carencias en infraestructura -mobiliario, básculas, brazaletes para el esfigmomanómetro, etcétera- para atender a pacientes con obesidad mórbida. La mayoría de los hospitales públicos de segundo y tercer nivel no cuenta con estos instrumentos. El número de grupos interdisciplinarios para la atención de estos pacientes es muy reducido.

4. Elevados costos de la atención. La cronicidad de estos padecimientos causa costos permanentes para el paciente y para los servicios de salud. La necesidad de una dieta correcta -la que con frecuencia es más costosa que las comidas a las que muchos pacientes tienen acceso- y de actividad física -que requiere de tiempo para su realización- son un gran obstáculo para el tratamiento, porque de una u otra manera implican costos para el paciente. Peor aún es la situación cuando el paciente requiere medicamentos que él mismo tiene que comprar. En las instituciones de salud la atención de estos pacientes es costosa. Requieren citas frecuentes y exámenes para evaluar la evolución de la obesidad y sus comorbilidades.
5. Ausencia de propuestas médicas que determinen cambios que el paciente perciba con claridad en el corto plazo. Tanto en pacientes con obesidad como en pacientes con síndrome metabólico -sin diabetes mellitus-el tratamiento no produce sensaciones de beneficio evidente. Esto contrasta con las grandes expectativas que suelen tener. La pérdida de peso lograda dista de ser la deseada. La reducción en cifras de tensión arterial y lípidos no necesariamente da bienestar y de hecho pueden presentarse efectos adversos. Distintos informes muestran que aun cuando el paciente reciba de manera puntual y gratuita sus medicamentos, la toma de los mismos disminuye más allá de lo aceptable con el paso del tiempo. La adherencia a los tratamientos es muy pobre.

6. Oferta de soluciones sin esfuerzo y productos milagro. El efecto nocivo de estas ofertas no se ha podido evaluar. Queda la impresión en los expertos que el daño es grande y que las estrategias publicitarias de estos productos suelen ser más efectivas que las utilizadas por el personal de salud para motivar a los pacientes. La evaluación cuidadosa de la metodología empleada por la industria para transmitir mensajes persuasivos conducirá a la implementación exitosa de campañas de prevención en la población.

7. Alta prevalencia de estos padecimientos. La elevada prevalencia de la obesidad y el síndrome metabólico hace necesario que los médicos generales, la mayoría de los médicos especialistas, los nutriólogos y los psicólogos estén familiarizados con las estrategias generales de su tratamiento. Esta competencia profesional -y peor aún el interés por parte de muchos de estos trabajadores de la salud- dista de ser una realidad y tiene que ser fomentada.

\section{Los problemas de los grupos vulnerables}

La obesidad se ha diseminado en toda la población. Es necesario, sin embargo, considerar las particularidades de ciertos grupos poblacionales que, por sus características y situación particular, son especialmente vulnerables a la obesidad y al síndrome metabólico; entre ellos, los niños, las mujeres, la población indígena y aquellos en situación de pobreza.

\section{Población infantil}

Es frecuente que en niños y adolescentes el sobrepeso pase inadvertido por sus padres e incluso por el médico que vigila su estado de salud y que aún cuando la obesidad exista, no se le dé suficiente importancia, debido 
a que erróneamente puede ser considerada como un estado transitorio e inherente a los primeros años de vida o incluso como normal.

La causa más frecuente de sobrepeso y obesidad es la combinación de las siguientes condiciones:

1. Presencia de variaciones genéticas responsables de la susceptibilidad a padecer obesidad.

2. Pérdida de balance entre la ingestión de energía y el gasto que se produce con el ejercicio. En nuestra sociedad, esta condición se ve frecuentemente favorecida por permitir que los niños consuman alimentos ricos en energía y grasas totales.

3. Falta de actividades deportivas de mediano y alto gasto energético de manera regular, es decir, tres o más veces por semana.

4. Realización de actividades sedentarias por más de tres horas al día: tareas escolares, televisión, juegos en computadora o electrónicos, etc.

Por otro lado, con frecuencia la mayoría de los médicos asumen erróneamente que el peso normal en el niño es el que se señala como promedio para la edad, sin considerar que el peso no se relaciona con la edad, sino con la estatura. Aunado a lo anterior, la educación escolar y extraescolar excluye la importancia de la obesidad, la manera de determinarla y sus consecuencias físicas, metabólicas, psicológicas y sociales, por lo que es fácil entender por qué aumenta progresivamente el peso que poco a poco convierte en obeso al niño o al adolescente.

Por ello es necesario transmitir a la sociedad la idea de que la obesidad es una enfermedad crónica, progresiva, no reversible por sí misma y que condiciona una serie de complicaciones que aumentan su gravedad conforme el peso se va incrementando. También es importante que la población conozca estrategias sencillas y efectivas para prevenir la obesidad. Por otra parte, se debe hacer énfasis en los problemas frecuentemente asociados al sobrepeso y a la obesidad, principalmente el exceso de producción de insulina con detrimento de su función (hiperinsulinemia con resistencia a la insulina), que es la base para el desarrollo de otras patologías secundarias como:

1. Alteración en los niveles de glucosa en sangre que llega a ocasionar diabetes no dependiente de insulina (diabetes tipo 2) a edades muy tempranas de la vida, frecuentemente desde el inicio de la pubertad. Es importante mencionar que la diabetes tipo 2 se llamaba diabetes del adulto por predominar en este grupo de edad; sin embargo, en los últimos años la diabetes tipo 2 ocurre cada vez a edades más tempranas, incluso cada vez es más frecuente en los niños.

2. Alteración de los lípidos circulantes con aumento de los triglicéridos y del colesterol transportado por lipoproteínas de baja densidad y disminución del transportado por lipoproteínas de alta densidad. Esto conduce a la producción de dislipidemia aterogénica, es decir, un estado que favorece la acumulación de grasa en las arterias y ocasiona ateroesclerosis, que es uno de los factores de más alto riesgo para la ocurrencia del infarto agudo del miocardio y de alteraciones vasculares en el sistema nervioso central (embolia, trombosis e infartos cerebrales).

3. Trastornos cardiacos relacionados con un incremento anormal de la cantidad de grasa abdominal, lo cual puede conducir al aumento del grosor del miocardio: hipertrofia ventricular izquierda.

4. Problemas respiratorios que se inician con menor capacidad para distender los pulmones (patrón restrictivo) y ocasionan menor oxigenación. Cuando la obesidad es acentuada, llega a producir períodos de falta de respiración durante el sueño con disminución de la concentración de oxígeno en la sangre y aumento de la concentración de bióxido de carbono, conocidos como apneas nocturnas con hipercapnea.

5. Depósito anormal de grasa en el hígado (esteatosis hepática) que puede ocasionar alteraciones funcionales progresivas como la cirrosis hepática no alcohólica.

6. Alteraciones ortopédicas por sobrecarga de las articulaciones, de la columna, la cadera, las rodillas, los tobillos y el arco del pie.

7. Defectos de postura al proyectar la cadera hacia adelante, flexionando caderas y rodillas, que progresan hacia desviaciones internas de las rodillas, externas de los tobillos y pérdida de la verticalidad de la columna vertebral.

8. Enfermedades irritativas de los pliegues cutáneos de axilas e ingles altamente susceptibles a infecciones por hongos como consecuencia de la sudoración profusa que caracteriza al exceso de grasa corporal.

Con base en lo anterior es necesario establecer estrategias adecuadas que permitan en su conjunto prevenir el sobrepeso y la obesidad para lo cual se plantean las siguientes medidas:

a) Reconocer que la obesidad es una enfermedad que puede padecerse a cualquier edad.

b) Informar a la comunidad sobre la forma de prevenirla. 
c) Capacitar a las familias y prestadores de servicios de la salud sobre la manera correcta de determinar el peso adecuado para la estatura.

d) Educar a la comunidad para llevar un estilo de vida saludable mediante el consumo de una dieta correcta, ${ }^{15}$ la realización de actividad física casi todos los días de la semana y reducción de actividades sedentarias. ${ }^{16}$

e) Promover que en las escuelas se expendan y se consuman alimentos bajos en energía, grasas, azúcares simples y sal y se destinen espacios adecuados para la realización diaria de actividad física durante por los menos 30 minutos.

f) Habilitar espacios públicos seguros y adecuados para realizar actividades deportivas o recreativas no sedentarias.

g) Regular la información sobre alimentos y bebidas industrializados en las horas de televisión y radio dedicadas al público infantil.

h) Vigilar la oferta de alimentos y bebidas industrializadas en cuanto al tamaño de las porciones y al contenido energético.

\section{Mujer}

La obesidad en la mujer tiene matices peculiares que comienzan a ser comprendidos. La mayor prevalencia de obesidad en las mujeres es producto de características biológicas y psicológicas así como de situaciones sociales.

Factores biológicos. En comparación con los hombres, el mayor porcentaje de masa grasa en las mujeres determina un gasto energético menor por kilogramo de peso, lo que hace más difícil perder el sobrepeso ganado. Los embarazos favorecen la obesidad, como lo sugieren los datos que muestran una asociación entre el número de embarazos y el desarrollo de obesidad. La menopausia tiene efectos desfavorables en la composición corporal que conduce a un aumento en la obesidad entre los 40 y 60 años, particularmente si no se hacen los ajustes necesarios en el balance energético.

Factores psicológicos. Las mujeres tienen mayor prevalencia de trastornos de la conducta alimentaria. Algunos estudios muestran mayor prevalencia de depresión y ansiedad entre las mujeres con obesidad. Estos factores psicológicos son a la vez causa y consecuencia de la obesidad.

Efecto del ambiente. El ambiente "obesogénico" puede tener mayor efecto en las mujeres porque las porciones que ingieren son habitualmente superiores a sus necesidades, ya que sus requerimientos energéticos son menores a los de los hombres por su fenotipo y su nivel de actividad.

Tratamiento de la obesidad en la mujer. Algunos hechos sugieren que la obesidad en las mujeres tiene efectos más desfavorables que en los hombres, particularmente en lo referente a riesgo cardiovascular. Por otra parte, algunos estudios han sugerido que los médicos tienen una actitud menos decidida y hasta reacia para iniciar el tratamiento de las comorbilidades de la obesidad y del síndrome metabólico en las mujeres, porque perciben que el riesgo que éstos representan en las mujeres es menor que el que perciben en los hombres.

Respecto a las consecuencias de la obesidad y del síndrome metabólico, se ha concluido en algunos análisis que las repercusiones psicosociales y económicas negativas de la obesidad son mayores entre las mujeres.

Hay evidencias de que las estrategias de tratamiento de la obesidad en las mujeres requieren tácticas puntuales de motivación a fin de promover una mejor adherencia y lograr el éxito terapéutico.

\section{Población indígena}

$\mathrm{Al}$ parecer, la población indígena tiene menor prevalencia de obesidad que el resto de la población. En un análisis de la ENSA 2000 se encontró que los adultos indígenas tienen menos obesidad ( $13.5 \%$ vs. $24.5 \%)$ y adiposidad central ( $31.1 \%$ vs. $43.1 \%$ ) que los no indígenas. La prevalencia de hipertensión y diabetes mellitus en adultos indígenas fue ligeramente menor a la del resto de la población; sin embargo, la probabilidad de carecer de un diagnóstico previo fue mayor. Esta situación hace a esta población muy vulnerable porque desconoce su enfermedad y por ello no busca atención oportuna; además, enfrenta una doble carga de enfermedad importante pues en estos grupos hay mayor prevalencia de enfermedades infecciosas, desnutrición y otras, en etapas previas de transición epidemiológica, que todavía representan un reto de salud pública. ${ }^{17}$ Por otra parte, la idiosincrasia de la población indígena respecto a la salud no es bien comprendida y por eso es probable que los esquemas habituales de prevención y tratamiento no sean aplicables a este grupo poblacional. Es necesario buscar esquemas aceptables para evitar el aumento en la prevalencia de estas enfermedades y sus comorbilidades en los indígenas.

\section{Población en situación de pobreza}

En el pasado, la pobreza se asoció tradicionalmente a problemas de desnutrición y a otras enfermedades por carencias. Sin embargo, hoy sabemos la doble carga de 
la enfermedad en población con carencias y que sufre inseguridad alimentaria. A la vez que existe desnutrición en esta población y deficiencia de nutrimentos específicos, la obesidad es cada vez más frecuente. Lo grave es que en ocasiones, en una misma familia coexisten la desnutrición y la obesidad pues puede haber una madre obesa con un hijo pequeño desnutrido.

La obesidad en la gente pobre ocurre por distintos mecanismos. Baste decir que los sobrevivientes de la desnutrición infantil son particularmente susceptibles a desarrollar obesidad, síndrome metabólico y otras enfermedades crónicas en la vida adulta. Esto es particularmente relevante en niños que sobreviven a la desnutrición y que posteriormente tienen crecimiento más acelerado.

Por otra parte, las dietas características de la población en situación de pobreza se caracterizan por ser de alta densidad energética y de baja densidad de nutrimentos, ya que aportan mayor volumen, saciedad y palatabilidad a un menor costo. Son dietas en las que predominan productos ricos en hidratos de carbono y lípidos, tradicionalmente no perecederos y escasea en las verduras y frutas. Además los alimentos de origen animal son de mayor costo y son perecederos. Las dietas de alta densidad energética pueden llegar a aportar energía en cantidades considerablemente superiores de las que se requiere.

Por otra parte, este grupo de población con frecuencia carece de oportunidades para llevar una vida activa. Esto, aunado a la desnutrición en la niñez y el tipo de dieta les da un alto riesgo de desarrollar obesidad y síndrome metabólico.

La población en situación de pobreza suele buscar atención médica para problemas de salud cuando están en estadios avanzados. Esto limita su calidad de vida y encarece los servicios de salud.

\section{Problemas de la salud mental en la obesidad y el sindrome metabólico}

El papel de la salud mental en el estudio y manejo de la obesidad no es sencillo. Se ha ido complicando conforme se ha profundizado en los factores psicológicos asociados al problema. En el centro de este modelo está el reconocimiento de un síndrome descrito por Stunkard en $1959,{ }^{18}$ un trastorno de conducta atípico en la nomenclatura moderna: el trastorno por atracón (TxA), el cual se acompaña frecuentemente de obesidad y se caracteriza por los siguientes datos: ${ }^{19}$

A. Atracones recurrentes caracterizados por:

- Ingerir una gran cantidad de alimentos.

- Tener la sensación de pérdida de control durante éstos.
B. Asociado con al menos tres de los siguientes:

- Comer más rápidamente de lo usual.

- Comer hasta estar incómodamente lleno.

- Comer cantidades exageradas sin tener hambre.

- Comer a solas por pena a que otros observen la cantidad y calidad de los alimentos ingeridos.

- Sentir culpa, coraje o tristeza después del atracón.

C. Malestar psicológico importante.

D. Atracones cuando menos dos días por semana por un lapso de seis meses.

E. No hay anorexia nervosa o bulimia nervosa, ni conductas compensatorias concomitantes.

El interés en esta alteración de conducta alimentaria ha aumentado debido a varios aspectos. Además de que esta entidad alimentaria tiene elevada asociación con el desarrollo de obesidad mórbida, los pacientes tienen menor calidad de vida ${ }^{20}$ y $30 \%$ de los que acuden a tratamiento de la obesidad cursan con criterios para el diagnóstico del TxA. ${ }^{21} \mathrm{Su}$ respuesta al tratamiento generalmente es menor cuando se compara la mejoría observada en pacientes obesos sin TxA. Sin embargo, lo más probable es que estos datos se deban a factores mediadores como los trastornos depresivos. Los pacientes obesos con TxA sufren más depresión/ ansiedad y tienen menor autoestima que aquellos sin TxA. ${ }^{22,23}$ Por otro lado, los pacientes obesos con TxA tienen mayor grado de impulsividad y mayor comorbilidad psicopatológica que los obesos sin TxA.

Respecto a la obesidad temprana, se han descrito varios antecedentes de negligencia y abuso infantil. Los niños desatendidos tienen 9.8 veces más probabilidades de volverse obesos. ${ }^{24}$ Los adultos que buscan tratamiento para obesidad tienen el antecedente de un incremento del cuádruple en la prevalencia de abuso sexual en la infancia y del doble en abuso no sexual. ${ }^{25}$

En el curso de varios trastornos mentales (e.g. la depresión atípica o los trastornos de ansiedad), es frecuente encontrar obesidad o al menos periodos de sobreingestión de energía. Quizás esto tiene que ver con mecanismos complejos radicados en circuitos cerebrales relacionados con la recompensa, la regulación afectiva o la respuesta al estrés. Existen opiniones a favor de la existencia de un modelo de "adicción a los alimentos" en algunos pacientes. ${ }^{26,27}$ Algunas personas que sufren de bulimia nervosa o de TxA relatan que sus atracones son desencadenados por estados emocionales adversos (disfóricos) y que esta conducta les trae una sensación de atenuación o mejoría emocional, que tiende a ser pasajera. De hecho, hoy se explora la psicología de la 
obesidad y de los trastornos psiquiátricos que se asocian con ella, en búsqueda de indicadores subyacentes de los fenotipos de conducta y sus endofenotipos. Recientemente se ha descrito que hay menor activación de la corteza dorsolateral prefrontal izquierda, área cerebral relacionada con la saciedad, ${ }^{28,29}$ en personas obesas en respuesta a una comida.

Las estrategias futuras para la prevención o el tratamiento de la obesidad tendrán que tomar en cuenta la identificación de sujetos susceptibles de desarrollar obesidad, el papel de los factores mantenedores que incluyan los aspectos psicobiológicos y psicopatológicos y el estudio de estrategias que prevengan las recaídas.

\section{Necesidad de comunicar lo que se sabe}

En el cuidado de la salud, la información puede hacer toda la diferencia. El tema de la obesidad ha trascendido el territorio de los investigadores, los académicos y los prestadores de servicios de salud, y parece ser tema del dominio de la población general. Esto se debe a que la obesidad se ha vuelto un problema de salud pública en nuestro país y empieza a ser percibido como tal por la población.

Actualmente, el acceso a la información es casi irrestricto. Cualquier persona interesada puede acceder en segundos a grandes cantidades de información. La obesidad es uno de los temas cuya información abunda. Desafortunadamente, no toda la información disponible es seria, verídica ni está científicamente respaldada. Esto ha generado gran desconcierto en la población al recibir información confusa, desarticulada y con frecuencia incorrecta.

Es obligación y compromiso de los prestadores de servicios de salud proporcionar a la población información con respaldo científico, actualizada, de fácil comprensión. Lo que se requiere es información práctica, que puntualice el problema de la obesidad, sus riesgos, sus estrategias de prevención y las consecuencias a nivel individual, familiar y social si no se toman las acciones necesarias para prevenirla o combatirla. La información que se proporcione a la población debe indicar el "qué", pero también el "cómo". No es útil decir a la población que debe mantenerse en un peso saludable; es necesario decirle cómo lograrlo. De nada servirá decirle que consuma más frutas y verduras; es necesario darle información de cómo optimizar sus recursos para adquirirlas baratas y frescas, con un óptimo contenido de nutrimentos; es necesario proporcionarle información de cómo prepararlas para que sean apetecibles y conserven sus propiedades.

A través de la educación se podrá transmitir lo que se sabe, ya que ésta permite que las personas piensen por sí mismas, tomen sus propias decisiones y se fijen sus propios objetivos de acuerdo con sus circunstancias.

La información que se dé a los pacientes no se debe concentrar toda al inicio del diagnóstico, tiene que ser continuada. Se debe hablar siempre de ganancias y no de pérdidas, en un lenguaje claro, que resuelva dudas sin dar por hecho que la gente comprende con tan sólo una instrucción. Se debe adaptar la información a la persona y no al revés.

Proporcionar al paciente una base teórica y práctica para la comprensión y para afrontar las consecuencias de la enfermedad le permitirá colaborar de forma activa con el equipo en todos los aspectos de su tratamiento.

Al transmitir lo que se sabe se promoverá que la situación sea aceptada, se le dará sentido a la información, se logrará moderar la angustia generada a partir del desconocimiento, desinformación o de ambos, se desarrollarán estrategias más adaptadas y saludables para afrontar eficazmente los síntomas y se podrá detectar la recurrencia de las recaídas.

\section{Necesidad de un nuevo modelo médico de atención}

El tratamiento de la obesidad obliga a cambios en el estilo de vida de los pacientes. Este proceso requiere una comunicación clara y convincente entre los sistemas de salud, la sociedad en general y los pacientes.

El modelo médico vigente y el ejercicio que deriva de él no han logrado detener el aumento de la prevalencia de la obesidad y del síndrome metabólico. Tanto la prevención como el tratamiento de la obesidad y el síndrome metabólico requieren estrategias complejas para las que no están preparados ni las instituciones, ni los trabajadores de la salud, ni los pacientes.

No es exagerado decir que se requiere una cultura de salud diferente. Los grandes logros de la medicina en el siglo XX dejaron la "impresión generalizada" en la sociedad de que la salud podría convertirse en un bien que sería generado fuera del individuo, a través de fármacos cada vez mejores y de intervenciones cada vez más avanzadas tecnológicamente. Los procesos crónicos degenerativos han hecho evidente el papel del ambiente y de las conductas de la sociedad y de los individuos en el desarrollo de estos padecimientos.

El trabajador de la salud tiene que convertirse en un promotor de conductas saludables. Para ello, frecuentemente, tendría que ser él mismo, el primero en cambiar su estilo de vida. El paciente tendrá que categorizar mejor sus prioridades para poder atender adecuadamente las necesidades de su cuerpo.

La construcción de un nuevo modelo de salud se vislumbra como un proceso lento que requiere procesos 
educativos en todos los niveles sobre el tema obesidad y sus consecuencias en la salud. Para la sociedad en general y los individuos en riesgo elevado o ya afectados por la obesidad y el síndrome metabólico se requiere la difusión de información y la elaboración de programas educativos permanentes. Los programas de estudio y todos los cursos de educación continuada, dirigidos a los trabajadores de la salud, deben incluir como elemento principal los temas relacionados con la prevención y tratamiento de estas entidades.

Es evidente la responsabilidad del gobierno como impulsor de la creación de un mejor modelo de atención de estos padecimientos. Son insuficientes las instalaciones y los grupos que trabajan en la investigación, prevención y tratamiento de la obesidad y el síndrome metabólico. Impulsar el desarrollo de los mismos bajo una adecuada coordinación es una necesidad urgente. La construcción de un ambiente más saludable tiene que ser una acción concertada, teniendo como principales agentes un gobierno preocupado y activo frente a una sociedad debidamente informada.

\section{Necesidad de un nuevo paciente}

La mayoría de los pacientes obesos saben lo que "se debe hacer" para bajar de peso. Han probado un sinfín de dietas; han intentado infinidad de veces hacer ejercicio, incluso han logrado bajar de peso en alguna ocasión, pero lo recuperan una vez que retoman sus hábitos de alimentación y sedentarismo. Y aún así, siguen buscando "un nuevo y mejor tratamiento" que les resuelva el problema de por vida.

Todos los profesionales de la salud sabemos que la obesidad es una enfermedad crónica recidivante, costosa, que requiere una educación constante y que se acompaña de instrucciones respecto a la modificación del estilo de vida, como cambios en la forma de comer, un plan de ejercicios, administración de medicamentos, saboteo familiar, frustraciones, mentiras, necesidad de magia, cansancio, abandono de tratamiento, etcétera.

Es por ello que además de la necesidad de generar un nuevo modelo de tratamiento que logre el objetivo, también se debe cambiar la mentalidad del paciente en relación con su problema y construir la infraestructura médica, emocional, familiar y social que permita que el paciente obeso logre lo que se propone.

Un paciente puede tener obesidad mórbida y sufrir las complicaciones médicas mayores que conlleva, pero no estar preparado para hacer un compromiso de cambio en su estilo de vida que lo conduzca a la reducción de peso. Por ello es importante que el profesional de la salud realice un interrogatorio dirigido a investigar si el paciente reconoce la necesidad de perder de peso, antes de asumir que se apegará al tratamiento. Los resultados del examen físico, de la historia clínica y las pruebas de laboratorio pueden ayudar para educar a los pacientes respecto de la necesidad de reducir su peso.

El profesional de salud también debe determinar el momento adecuado para que el paciente pierda peso. La pérdida de peso requiere concentración y esfuerzo sostenidos; sin embargo, estos pueden ser estropeados por la ocurrencia de estresores en la vida, como puede ser un cambio de trabajo, problemas económicos, enfermedad de algún familiar, entre otros. ${ }^{30} \mathrm{Si}$ el paciente siente que es incapaz de apegarse al tratamiento, se debe esperar hasta que sus circunstancias de vida mejoren. Si esto sucede, la meta inmediata de tratamiento es la prevención de mayor ganancia de peso en lugar de la inducción de la pérdida de peso.

Para evaluar la disposición al cambio que tiene cada paciente, es necesario identificar si se manifiestan ambivalencias y resistencias ante el tratamiento o si el paciente no está motivado. Prochascka, DiClemente y Norcross ${ }^{31}$ propusieron un modelo de los niveles de motivación en los pacientes con trastornos de alimentación (cuadro II).

Hay que recordar que las enfermedades crónicas deben ser tratadas con paciencia y no sólo prescribir un medicamento y esperar que éste haga efecto. Estamos frente a individuos que tienen un padecimiento incurable, con recidivas frecuentes, con aparición de complicaciones y con un tratamiento que no convence, y no por ser un planteamiento erróneo, sino porque el cambio en el estilo de vida es responsabilidad total del paciente y de su familia. Los cambios en la alimentación

\section{Cuadro II \\ Modelo de los niveles de motivación PARA SEGUIMIENTO DEL TRATAMIENTO EN LOS PACIENTES OBESOS}

\begin{tabular}{ll} 
I.Precontemplación & No hay intención de cambio. \\
\hline 2. Contemplación & $\begin{array}{l}\text { Hay conciencia de los problemas, se piensa en ellos, } \\
\text { pero no hay intención de cambiarlos. }\end{array}$ \\
\hline 3. Preparación & $\begin{array}{l}\text { Existe la intención de cambiar, pero no lo ha hecho } \\
\text { en el último año. }\end{array}$ \\
\hline 4.Acción & $\begin{array}{l}\text { Se cambian conductas, actitudes, experiencias o el } \\
\text { entorno para superar el problema. }\end{array}$ \\
\hline 5. Mantenimiento & $\begin{array}{l}\text { Ya se han modificado las conductas, se previenen } \\
\text { recaídas y se consolidan los logros. }\end{array}$
\end{tabular}

Fuente: Referencia 31 
y en la actividad física son la clave del éxito. ¿Pero quién logra perder peso y mantener el éxito?

Se ha intentando identificar las características de pacientes que logran el objetivo de bajar de peso y más aún de mantenerlo. Los que han tenido éxito al inicio del tratamiento atribuyen el sobrepeso a sus hábitos de alimentación; tienen pobre calidad de vida, menos trastornos psicopatológicos como síntomas depresivos, trastornos en la conducta de alimentación y desinhibición. Además, han hecho menos intentos de bajar de peso, tienen una motivación intrínseca, más restricción cognitiva, mayor autoeficacia, más insatisfacción corporal, mayor riesgo de enfermedad coronaria y niveles altos de colesterol. Una vez que se logran los resultados se observa que a lo largo del tratamiento y en el momento de la valoración final, los pacientes que tuvieron éxito tienen mayor pérdida de peso inicial, realizan más actividad física, son menos propensos a comer por estímulos externos, son menos exigentes al valorar los cambios en el peso y consumen más fibra. ${ }^{32-37}$

Esto nos lleva a la necesidad de reconocer, diagnosticar, dar tratamiento y seguimiento al paciente obeso considerando los puntos mencionados, para generar nuevas estrategias de intervención y crear "nuevos pacientes".

Para disminuir las resistencias que presentan los pacientes obesos, es importante lo siguiente: ${ }^{10}$

1. Incrementar la empatía entre médico y paciente.

2. Fomentar la conciencia de enfermedad, tanto en el paciente como en su familia.

3. Reconocer las consecuencias positivas y negativas del tratamiento.

4. Reconocer las inconsistencias previas entre los objetivos y las conductas de los pacientes en los tratamientos previos.

5. Estimular al paciente para que adquiera un papel activo en su cambio de conducta.

Aún más, independientemente del tratamiento que se haya elegido para tratar la obesidad, la forma en que el profesional de la salud discute el tratamiento con los pacientes puede tener un efecto profundo en el resultado. En ningún tratamiento es más evidente la necesidad de empatía y de expectativas realistas que en el desafío de mantener la pérdida de peso. Incluso, el mantenimiento del peso requiere un conjunto de habilidades diferentes a las utilizadas durante la pérdida de peso, ya que requiere tanto o más esfuerzo que dicha pérdida, pero tiene menos gratificación o satisfacción. ${ }^{33}$

Hacer énfasis en los beneficios de las conductas de pérdida de peso, disminuir los pretextos para realizar ejercicio, incrementar la seguridad de poder elegir ali- mentos bajos en grasa, mejorar las herramientas para utilizar etiquetas de alimentos, educar a los pacientes sobre las expectativas del tratamiento, describir las demandas de conducta del programa (p. ej. automonitoreos de comida y ejercicio), así como discutir los riesgos y beneficios del procedimiento, son estrategias que se pueden utilizar en la preparación del paciente obeso para iniciar un nuevo estilo de vida. ${ }^{34}$

\section{Consecuencias de la desinformación}

y de las malas prácticas

No es difícil visualizar las consecuencias de la desinformación poblacional con respecto a la nutrición adecuada y las malas prácticas que ésta genera. Respecto a las características nutricias de los diferentes alimentos y las conductas compensatorias para el control de peso, en nuestra población prevalecen ideas sin ningún sustento e incluso, sin sentido común. Todo ello sucede, por un lado, en pacientes con trastornos alimentarios caracterizados por la búsqueda de la delgadez extrema (i.e. anorexia nervosa, bulimia nervosa y algunos trastornos alimentarios no especificados) y por el otro, en pacientes obesos donde frecuentemente están mal informados acerca de dietas "maravillosas" para disminuir su peso, así como la falta de capacidad para discernir sobre las mejores formas de controlar su obesidad. De hecho, la meta que persigue el paciente al disminuir de peso es sentirse satisfecho consigo mismo y por ende, mejorar su autoestima, dejando en segundo lugar los beneficios en su salud, los cuales la mayoría de ellos conocen en forma superficial.

Es frecuente que estos pacientes desarrollen conductas compensatorias alteradas, por ejemplo, provocación de vómito o el uso de laxantes o diuréticos, como un recurso desesperado para disminuir la ansiedad que deriva de haber comido en forma exagerada. Más aún, muchos pacientes que desarrollan anorexia o bulimia nervosas tienen entre sus antecedentes el haber sido obesos previamente.

Es por ello que desde hace varios años, como parte inicial insustituible de cualquiera de las rutas de tratamiento vigentes, en algunas instituciones se practica la psicoeducación, proceso que facilita enormemente el inicio del cambio de conductas alteradas a la manera de los procesos descritos por Prochaska y DiClemente ${ }^{31}$ para el tratamiento del tabaquismo. Esta psicoeducación se lleva a cabo con una estrategia que favorece la comprensión del paciente y de sus familiares acerca de las características de su padecimiento y la manera de participar para resolverlo. No se trata de "clases magistrales", sino más bien de diálogos abiertos y en lenguaje sencillo. Se dan ejemplos concretos, pro- 
yecciones imaginativas e incluso de la paradoja, para dejar en claro un sustento razonable para cada una de las ideas que les proponemos. Tras un proceso de cinco sesiones psicoeducativas, es frecuente que ocurra una disminución sintomática en nuestros casos o una mejor disponibilidad para el trabajo en equipo que hacemos con la familia. El uso de las réplicas de alimentos ayuda a los nutriólogos a aclararle al paciente y a sus familiares sobre el tamaño real de las raciones de alimentos.

A los pacientes que incurren en atracones, se les hace comprender la diferencia entre la sensación de saciedad y la sensación de estar lleno, lo cual es muchas veces esclarecedor para ellos. Posteriormente se les instruye sobre algunas prácticas que promueven la saciedad y se les pide llevar un registro alimentario en donde, además de anotar su ingestión de alimentos, sean de forma programada o de atracones; se les instruye para registrar las condiciones desencadenantes de estos últimos. Esto se debe a que la mayoría de nuestros pacientes con bulimia nervosa o trastorno por atracón asociado a obesidad utilizan el atracón como una táctica para la distensión de estados emocionales alterados, a la manera de algunas adicciones.

Aunque el trabajo por venir es arduo, desde nuestro punto de vista, la transmisión de información sustentada y accesible a los pacientes obesos y a sus familiares a través de la psicoeducación interactiva, así como el esclarecimiento psicoeducativo de la asociación de estados emocionales adversos y el inicio de los atracones, y la detección del papel que estos atracones tienen en la atenuación de las emociones adversas que los desencadenan, son de primera importancia en cualquier estrategia destinada a detectar y tratar la obesidad.

\section{Investigación en la obesidad}

La investigación sobre las causas, los modelos de atención, la prevención y el tratamiento de esta entidad debe ser considerada como una inversión en salud de altísima prioridad.

Distintas áreas de investigación en obesidad requieren un estudio cuidadoso y un presupuesto suficiente que garantice resultados confiables. Tales áreas son:

1) Investigación sobre las causas de la enfermedad
a) Biológicas
b) De conducta
c) Culturales
d) Sociales
e) Antropológicas

2) Investigación sobre modelos de atención
a) Primer nivel
b) Segundo nivel
c) Tercer nivel

3) Investigación sobre estrategias de tratamiento
a) Primer nivel
b) Segundo nivel
c) Tercer nivel/ En distintas especialidades médicas

4) Investigación sobre estrategias y modelos de prevención
a) Individuales
b) Familiares
c) Sociales
d) Gubernamentales (federales y estatales)
e) Primer nivel
f) Segundo nivel

5) Investigación sobre los impactos sociales y económicos derivados de la atención de pacientes con enfermedades crónico degenerativas

El enfoque actual de investigación debe ser "global" y no reducido a una dicotomía. El análisis de aspectos genéticos, geodemográficos, de balance energético, inflamatorios, neurohormonales, antropométricos, clínicos y sociomédicos, deben ser el soporte del "juicio clínico moderno" para una mejor práctica de la medicina. La investigación debe hacerse en un sentido de "comunicación horizontal/vertical -matricial-" entre todas las áreas involucradas y no únicamente en sentido vertical (separadas) como tradicionalmente se desarrolla en muchos centros. Los nuevos retos de la salud en el tema de obesidad y de síndrome metabólico y sus consecuencias se deben confrontar en forma integral.

En las áreas de investigación propuestas es necesario establecer mecanismos de evaluación y seguimiento que evalúen la eficacia y el costo-beneficio de los resultados en cada una de ellas.

\section{Modelo de coordinación entre los Institutos y sus tareas: conclusiones y propuestas.}

Los Institutos Nacionales de Salud han surgido como respuesta a una necesidad de la salud pública y el desarrollo de la medicina en México. La colaboración entre ellos ha tenido lugar a lo largo de su historia como producto también de necesidades y proyectos concretos. 
La obesidad y el síndrome metabólico, como problemas de salud muy prevalentes, afectan a pacientes que son objeto de asistencia y líneas de investigación en cada uno de los Institutos y Hospitales de Alta Especialidad. Estamos conscientes de que existe colaboración entre médicos e investigadores de los distintos Institutos en la asistencia y la investigación sobre la obesidad y el síndrome metabólico. Pretender regular estas colaboraciones surgidas de la realidad no sería conveniente. Sin embargo, fomentarlas y favorecerlas sería deseable. Proponemos para ello las siguientes acciones:

I. Organizar jornadas anuales por el Grupo Académico para el Estudio la Prevención y el Tratamiento de la Obesidad y Síndrome Metabólico de la CCINSHAE donde se reúnan médicos, nutriólogos, psicólogos e investigadores interesados en el estudio de la obesidad y el síndrome metabólico para fomentar la colaboración entre los Institutos y compartir experiencias exitosas en la prevención, detección y tratamiento de estos problemas. Se iniciará en forma modesta y se ampliará gradualmente su alcance.

Características del evento:

1. Las jornadas deberán estar regidas por un espíritu de diálogo para la construcción de propuestas, acuerdos y colaboraciones.

2. Los participantes deberán laborar en los Institutos Nacionales de Salud en los Hospitales Federales de Referencia o en los Hospitales de Alta Especialidad que dependen de la CCINSHAE.

3. El evento será convocado por el Titular de la CCINSHAE.

4. Las conclusiones y propuestas derivadas de estas reuniones serán parte fundamental de un documento que será turnado a los directores de los Institutos, al Secretario de Salud, a otros Secretarios de Estado y a legisladores.

5. La agenda del evento será propuesta por los miembros del Grupo Académico para el estudio, prevención y tratamiento de la obesidad y el síndrome metabólico de la CCINSHAE. Estos temas versarán sobre las siguientes líneas:

- Modelos de atención

- Estrategias de prevención y tratamiento

- Prioridades en la investigación clínica y básica

- Coordinación entre los Institutos Nacionales y vinculación

II. Realizar una campaña de difusión para fomentar el autodiagnóstico; con objeto de que el paciente conozca su IMC y su cintura y lo que esto significa.
Su objetivo principal será iniciar la adopción de un lenguaje común entre todos los pacientes y trabajadores de la salud de los INSHAE.

Todo paciente de los INSHAE-sin importar el motivo de su consulta- deberá conocer:

- Su IMC y cómo se calcula

- El perímetro de su cintura

- $\quad$ Lo que su IMC y su cintura significan en su estado de salud

- Lo que su IMC y su perímetro de cintura significan en los costos de su atención médica para las instituciones de salud, pero sobre todo para él mismo y para su familia

- La evolución de su IMC y del perímetro de cintura a lo largo de su seguimiento

III. Realizar una campaña de alimentación correcta y actividad física.

El objetivo principal será hacer consciente al individuo de que los alimentos que come y la actividad física que realiza tienen un efecto directo en la génesis del sobrepeso y la obesidad. En esta campaña se darán mensajes breves y concretos aplicables a la población general. Se dará información de cómo alimentarse mejor y con menor costo; cómo aumentar la actividad física de una manera práctica. Se utilizarán estrategias publicitarias exitosas, similares a las utilizadas por la industria alimentaria contratando profesionales de la comunicación en estrecha coordinación con el grupo de expertos. A continuación se presentan algunos ejemplos del rubro de alimentación correcta:

- ¿Sabías que hay una gran variedad de vegetales verdes de los que puedes comer cuanto quieras?

- ¿Sabías que las bebidas que contienen energía son una de las principales causas del sobrepeso y la obesidad? ¡Bebe agua!

- ¿Sabías que seleccionando los alimentos adecuados puedes comer mayor cantidad de alimentos y obtener menos energía? Aquí te decimos cómo.

- ¿Sabías que cualquier platillo puede aumentar su capacidad para producir sobrepeso y obesidad si se elabora con un exceso de grasa?

Para los rubros II y III la población objetivo será la de los Institutos Nacionales de Salud, Hospitales Federales de Referencia y Hospitales de Alta Especialidad e incluye a su personal administrativo, de intendencia y de salud, y a los pacientes y sus familiares. 
Es necesario que los procesos de mercadeo social se elaboren en forma profesional para que las campañas de promoción de la salud y de prevención que se lancen al interior de los institutos sean eficaces y sensibilicen incluso a la población que no tiene conciencia del problema. Será responsabilidad de este grupo de expertos elaborar una lista con los contenidos principales de las campañas y revisar los mensajes desarrollados por los expertos en mercadeo social. Las campañas al interior de los institutos estarán orientadas a sensibilizar a la población del problema y a obtener indicadores que permitan cuantificar la situación inicial y el éxito de las campañas.

IV. Identificar las necesidades prioritarias en la investigación básica y clínica por el grupo de expertos en obesidad y síndrome metabólicos de los INSHAE en su estudio y tratamiento y difundir esta visión entre los distintos grupos de investigadores de los institutos y de todas las instituciones con las que los institutos tienen estrecha colaboración: universidades, instituciones de salud, industria farmacéutica, industria alimentaria, etcétera. La investigación deberá estar encaminada a la acción.

\section{En concreto:}

Los INSHAE requieren un plan maestro acerca de la obesidad y el síndrome metabólico. La vinculación entre los CINSHAE, particularmente en las áreas de investigación y programas al interior de las instituciones, es un área de oportunidad para la búsqueda de respuestas que permitan encontrar una solución al problema. El siguiente paso sería la vinculación con el primer y segundo niveles de atención y con otras Secretarías e instancias que compartan metas comunes. Además de la investigación se necesitan acciones concretas para limitar el problema y posteriormente abatirlo. El costo de no hacer nada es demasiado alto para las instituciones, para las familias y para los individuos.

Entre los factores determinantes de la carga de enfermedad en el mundo y en México, la Organización Mundial de la Salud ha reconocido a aquellos relacionados con el estado nutricio y la actividad física como algunos de los más importantes. Estos tienden a producir una gran carga de enfermedad a través de la obesidad y el desarrollo de enfermedades crónicas como la diabetes tipo 2, las enfermedades cardiovasculares y las dislipidemias. Sólo cuando exista una respuesta social organizada que involucra todos los sectores de la sociedad para lograr los cambios necesarios en los distintos los niveles, se logrará combatir con éxito esta carga. El sector salud es pilar en esta tarea. Lo anterior no depende de falta de comprensión sobre los determinantes o de falta de tecnología para tratamientos, sino de una buena traducción del conocimiento en información para la población.

\section{Referencias}

I.World Health Organization. Obesity: preventing and managing the global epidemic. Report of a WHO Consultation on Obesity.WHO/NUT/ NCD/98.I. Geneva, Switzerland:WHO, 1998.

2. OMS:Alberti FGMM, Zimmet PZ, for the WHO Consultation: Definition, diagnosis and classification of diabetes mellitus and its complications. Part I:Diagnosis and classification of diabetes mellitus, provisional report of a WHO consultation. Diabet Med 1998; I5:539-553

3.ATP III. Expert panel on detection, evaluation and treatment of high blood cholesterol in adults. Executive summary of the third report of the National Cholesterol Education Program (NCEP) Expert panel on detection, evaluation and treatment of high cholesterol.JAMA 200I;285:2486-2497 4. IDF adultos. The International Diabetes Federation:The IDF consensus worldwide definition of the metabolic syndrome, 2005

Disponible en: http://www.idf.org/webdata/docs/Metac_syndrome_def.pdf 5. IDF niños. Zimmet P,Alberti G, Kaufman F, et al. The metabolic syndrome in children and adolescents. Lancet 2007;369:2059-206 I

6. Olaiz-Fernández G, Rivera-Dommarco J, Shamah-Levy T, Rojas R, Villalpando-Hernández S, Hernández-Avila M, et al. Encuesta Nacional de Salud y Nutrición 2006. Cuernavaca, México: Instituto Nacional de Salud Pública, 2006.

7. Lopez AD, Mathers CD, Ezzati M, Jamison DT, Murray CJL. Global and regional burden of disease and risk factors, 200I: systematic analysis of population health data. Lancet 2006; 367: I747-I757.

8. Velázquez O, Rosas M, Lara Ea, Pastelin HG, Sánchez CC, Grupo ENSA2000, et al. Prevalencia en interrelación de las enfermedades crónicas no transmisibles y factores de riesgo cardiovascular en México. Arch Cardiol Mex 2003; 73: 62-77.

9. Lara A, Rosas M, Pastelín G,Aguilar C,Attié F, Velásquez MO. Hipercolesterolemia e hipertensión arterial en México: Consolidación urbana actual con obesidad, diabetes y tabaquismo. Arch Cardiol Mex 2004; $74: 231-245$

10. Feinstein AR. The problem of Cogent Subgroups:A Clinicostatistical Tragedy.J Clin Epidemiol 1998; 51: 297-299.

II. Rosas M, Lara EA, Pastelín G,Velázquez O, Martinez-Reding J, Méndez A, et al. Re-encuesta Nacional de Hipertensión Arterial: Consolidación Mexicana de los Factores de riesgo Cardiovascular. Cohorte Nacional de Seguimiento.Arch Cardiol Mex 2005; 75: 96-III.

12. Rosas M. Definición de Síndrome Metabólico: La torre de Babel.Arch Cardiol Mex 2005; 75: 230-233.

13. Frenk J. Bridging the divide: global lessons from evidence-based health policy in Mexico. Lancet 2006; 368: 954-96I.

14. Listado de Oficiales Mexicanas de la Secretaría de Salud. [Consultado el 10 de enero de 2008]. Disponible en: http://www.salud.gob.mx/ unidades/cdi/nomssa.html.

15. Norma Oficial Mexicana NOM-043-SSA2-2005, Servicios básicos de salud. Promoción y educación para la salud en materia alimentaria. Criterios para brindar orientación. 23 ene, 2006.

16. Joint WHO/FAO Expert Consultation on Diet, Nutrition and the Prevention of Chronic Diseases. Geneva:WHO/FAO, 2002. Diet, nutrition and the prevention of chronic diseases: Report of a joint WHO/FAO Expert Consultation,WHO technical report series; 916. Ginebra, 2003. 
[Consultado el 21 de enero de 2008]. Disponible en: http://whqlibdoc. who.int/trs/WHO_TRS_916.pdf (Consultado 21-enero-2008)

17. Barquera S, Carrión C, Campos I, Espinosa J, Flores M, Olaiz G. Hipertensión (HT) y diabetes mellitus (DM) en indígenas mexicanos: Inequidad en la doble-carga de la enfermedad. Congreso Latinoamericano de Nutrición, Noviembre 2007, Florianopolis Brasil. (Ns 0I22). 18. Stunkard AJ. Eating patterns and obesity. Psychological Bulletin 1959;33, 284-294.

19. López-lbor,Aliño JJ,Valdés-Miyar M, coord. Manual diagnóstico y estadístico de los trastornos mentales. Barcelona: Masson, 2002. 20. De Zwaan M, Mitchell JE, Howell LM, Monson N, Swan-Kremeier L, Roerig JL, et al. Two measures of health-related quality of life in morbid obesity. Obes Res 2002;10(1I):1|43-1151.

21. de Zwaan M. Binge eating disorder and obesity. Int J Obes Relat Metab Disord 200I; 25 Suppl I:S5I-S55.

22. Jirik-Babb P, Geliebter A.Comparison of psychological characteristics of binging and nonbinging obese, adult, female outpatients. Eat Weight Disord 2003;8(2): 173-I77.

23. Fontenelle LF, V tor Mendlowicz M, de Menezes GB, Papelbaum M, Freitas SR, Godoy-Matos A, et al. Psychiatric comorbidity in a Brazilian sample of patients with binge-eating disorder. Psychiatry Res 2003; 15; I I9(I-2):189-194.

24. Lissau I, Sørensen TIA. Parental neglect during childhood and increased risk of obesity in young adulthood. Lancet 1994;343:324-327.

25. Felitti V]. Childhood sexual abuse, depression, and family dysfunction in adult obese patients: a case control study. South Med J 1993;86(7):732-736. 26. Finlayson $\mathrm{G}$, et al. Liking vs. wanting food: importance for human appetite control and weight regulation. Neurosc Biobehav Rev 2007;31:987-1002.

27. Devlin MJ: Is there a place for obesity in DSM-V? Int J Eat Disord 2007;40:S83-S88.

28. Duc Son NT Le, et al. Less activation in the left dorsolateral prefrontal cortex in the reanalysis of the response to a meal in obese than in lean women and its association with successful weight loss. Am J Clin Nutr 2007;86:573-579.

29. Le NTDS, Pannacciulli N, Chen K, et al. Lesser activation of the left dorsolateral prefrontal cortex in response to a meal: a feature of obesity, Am J Clin Nutr 2006;84:725-73I.

30. Wadden TA, Letizia KA. Predictors of attrition and weight loss in patients treated by moderate and severe caloric restriction. In:Wadden TA, Vanltallie TB, eds. Treatment of the Seriously Obese Patient. New York, NY: Guilford Press 1992:383-4I0.

31. Prochascka JO, DiClemente CC, Norcross JC. In search of how people change:Applications to addictive behavior.Am Psychologist 1992;4: I02-I I4. 32. Wiltink J, Dippel A, Szczepanski M, Thiede R, Alt C, Beutel ME. Longterm weight loss maintenance after inpatient psychotherapy of severely obese patients based on a randomized study: predictors and maintaining factors of health behavior.J Psychosom Res 2007;62(6):691-698.

33.Wadden TA. What characterizes successful weight maintainers? In:Allison D, Pi-Sunyer F, eds. Obesity Treatment: Establishing Goals, Improving Outcomes, and Reviewing the Research Agenda. New York, NY: Plenum Publishing Corp, 1995: 103-III.

34. Krummel DA, Semmens E, Boury J, Gordon PM, Larkin KT. Stages of change for weight management in postpartum women.J Am Diet Assoc 2004;104(7): I 102-I 108.

35. Teixeira PJ, Palmeira AL, Branco TL, Matins SS, Minderico CS, Barata JT et al. Who will lose weight? A reexamination of predictors of weight loss in women. Int J Behav Nutr Phys Act 2004;2(I): 12.

36. Texeira PJ, Going SB, Houtkooper LB, Cussler EC et al. Exercise motivation, eating and body image variables as predictors of weight control. Med Sci Sports Exerc 2006;38: 179-188.

37. Roberts $A$. What are the characteristics of overweight and obese patients who achieve weight loss and what factors are most helpful? A quantitative and qualitative study of patients and interventions in a rural general practice.) Hum Nutr Diet 1999; 12 (Suppl I):20-27. 